\title{
Flexural-Torsional Flutter and Buckling of Braced Foil Beams under a Follower Force
}

\author{
Manuel Ferretti, ${ }^{1,2}$ Francesco D'Annibale, ${ }^{1,2}$ and Angelo Luongo ${ }^{1,2}$ \\ ${ }^{1}$ International Research Center on Mathematics and Mechanics of Complex Systems, University of L'Aquila, 67100 L'Aquila, Italy \\ ${ }^{2}$ Department of Civil, Construction-Architectural and Environmental Engineering, University of L'Aquila, 67100 L'Aquila, Italy
}

Correspondence should be addressed to Angelo Luongo; angelo.luongo@univaq.it

Received 30 May 2017; Revised 13 August 2017; Accepted 24 August 2017; Published 12 October 2017

Academic Editor: Salvatore Caddemi

Copyright (C) 2017 Manuel Ferretti et al. This is an open access article distributed under the Creative Commons Attribution License, which permits unrestricted use, distribution, and reproduction in any medium, provided the original work is properly cited.

The flutter and buckling behavior of a cantilever foil beam, loaded at the tip by a follower force, are addressed in this paper. The beam is internally and externally damped and braced at the tip by a linear spring-damper device, which is located in an eccentric position with respect to beam axis, thus coupling the flexural and torsional behaviors. An exact linear stability analysis is carried out, and the linear stability diagram of the trivial rectilinear configuration is built up in the space of the follower load and spring's stiffness parameters. The effects of the flexural-torsional coupling, as well as of the damping, on the flutter and buckling critical loads are discussed.

\section{Introduction}

Dynamic stability of elastic systems loaded by nonconservative and configuration-dependent loads, such as follower forces $[1,2]$, has been thoroughly investigated by many researchers in the last century [3-10]. Some experimental evidences, proving the existence of such a kind of actions in the real world applications, are available, for example, in [10-12] and in the critical review [13], notwithstanding the fact that the engineering world is still suspicious of their existence and physical meaning. However, the effects on dynamic stability due to the presence of follower forces are very important in several engineering branches, such as in aerospace $[10,14,15]$, in flexible pipes conveying fluid [16-18], and in vehicle brakes $[19,20]$.

Researchers have devoted great attention in the last years to the so-called Beck's beam (see, e.g., $[4,5,13]$ ), namely, a cantilever beam loaded at the tip by a follower force (i.e., a force which keeps its direction tangential to the centerline), and, eventually, in the presence of conservative loads, and/or of distributed (internal and external) as well as lumped forms of damping. This structure indeed represents a paradigmatic system for the comprehension of stability issues in onedimensional nonconservative systems; in fact, the loss of stability may happen either by divergence, in the presence of conservative loads or lumped springs, or by flutter, also said to be Hopf bifurcation in Dynamical System Theory, depending on the mechanical properties of the structure [2124]. Moreover Beck's beam is also able to show one of the most amazing phenomena, occurring in the dynamical behavior of elastic systems loaded by follower forces, namely, the destabilizing effect of damping, or the "Ziegler Paradox"; see, for example, [3-6, 25-27]. It occurs when a vanishingly small and positive-definite damping is added to such a system, entailing a finite reduction of the flutter critical load with respect to that of the undamped system. Several contributions can be found in the literature, which are devoted to giving an explanation of this occurrence and to present different case studies, pointing out the phenomenon; among the others, the reader can refer to $[8,9,24,28-33]$.

In most of the previously cited papers a planar Beck's beam, under in-plane loads, is considered, whose trivial rectilinear configuration loses its stability in the same plane. However, when considering spatial beams, the loss of stability can occur out of that plane due to a flexural-torsional mechanical coupling. This phenomenon is well known in buckling analysis of spatial structures, such as thin-walled members, which, indeed, can exhibit a flexural-torsional Eulerian bifurcation, when subjected to conservative forces $[34,35]$. 
The flexural-torsional coupling may become important when issues relevant to dynamic stability are addressed. In this framework classical examples can be found mainly in aerospace engineering, for example, when the flutter behavior of a wing, immersed in a gas flow, namely, under nonconservative and velocity-dependent loads, is considered $[5,36]$. Other examples, when configuration-dependent loads act, can be found in [37], where the flutter instability of a cantilever beam containing a tip mass, subjected to a transverse follower force at the tip, and in the presence of airflow, is addressed; in [38], where the lateral-torsional stability of deep cantilever beams loaded by a transverse follower force at the tip, is studied; in [39], where the lateral stability of a slender beam, under a transverse follower force is addressed; in [21], where the flexural-torsional bifurcations of a cantilever beam under the simultaneous action of a nonconservative follower force and a conservative couple at the free end, have been analyzed; and finally in [40], where the bending-torsional flutter analysis of a cantilever, containing an arbitrarily placed mass, under a follower force and airflow, is analyzed. Remarkably, in the greatest part of the previous papers, a foil beam, namely, a beam for which one of the two inertia moments is much larger than the other, is considered as the mathematical model of aircraft's wing.

This paper is framed in the scenario illustrated above. Indeed, to the best of author's knowledge, there are no contributions in the literature addressing the flutter and buckling analyses of a spatial Beck's column, so that the present work is a first step toward the study of the problem. To this end, reference will be made to the simplest model as possible, namely, a clamped-free foil beam, loaded at the tip by a tangential follower force, internally and externally damped. Moreover, in order to couple the flexural and torsional behavior even in the linear range, it is assumed that the beam is braced at the tip by a linear spring-damper device, which is orthogonal to the axis line, and eccentric with respect to it.

The paper is organized as follows. In Section 2 the equations of motion of the model are presented. In Section 3 the eigenvalue problem is addressed and an exact linear stability analysis is carried out, both in the presence and in the absence of damping. In Section 4 a numerical analysis is developed, and the linear stability diagrams are built up in the space of the follower load and spring's stiffness parameters and for different damping coefficients. Finally, in Section 5 some conclusions are drawn.

\section{Model}

The foil beam is modeled as one-dimensional, inextensible and twistable, polar continuum (see, e.g., [41]), embedded in a three-dimensional space spanned by the unit vectors $\overline{\mathbf{a}}_{x}, \overline{\mathbf{a}}_{y}$, and $\overline{\mathbf{a}}_{z}$ (Figure 1). It is assumed that $z$ is the strong and $y$ the weak axis of the cross-section, that is, the inertia moments are $J_{z} \gg J_{y}$; consistently, the $x y$-plane is here referred to as the strong plane, while $x z$-plane is the weak one. If forces in the strong plane are smaller or, at most, comparable with those acting in the weak plane, then the beam can be considered unflexurable in the strong plane,

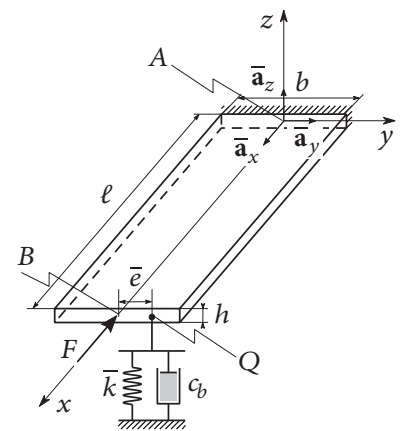

FIGURE 1: Foil beam viscoelastically braced under follower force.

and the relevant bending moment $M_{z}$ is a reactive stress. Concerning torsional stiffness, it is of the same order of magnitude of the weak bending stiffness, as it happens for compact cross-sections. Therefore, if torsional moments $M_{x}$ are smaller than the bending moment $M_{y}$, torsion is also negligible, so that the foil beam behaves as a planar beam in bending. Throughout the paper it is assumed that the torsional moment is comparable with the bending moment, so that the foil beam behaves as a flexural-torsional beam.

The beam is clamped at $A, s=0$, and free at $B, s=$ $\ell$, where $s$ is the abscissa spanning the points of the axis line; its flexural and torsional stiffness are $E J_{y}$, and $G J_{x}$, respectively, with $E$ and $G$ being the elastic and tangential moduli; moreover, $m$ is the mass per unit length and $I_{x}$ is the rotational moment of inertia. The beam is loaded at $B$ by a follower force, having intensity $F$, which keeps its direction parallel to the tangent to the centerline at $B$. The internal dissipation is assumed to be ruled by a linear Kelvin-Voigt law, whose flexural and torsional viscosity coefficients are $\eta$ and $\zeta$, respectively. An external viscous dissipation (e.g., due to the surrounding air) is also taken into account by a uniform distribution of linear dashpots, whose flexural and torsional viscosity coefficients are $c_{t}$ and $c_{r}$, respectively. Finally, the beam is viscoelastically braced at $B$ by a linear spring-damper device, having elastic and viscosity coefficients $\bar{k}$ and $c_{b}$, respectively; it is located at the point $Q=(\ell, \bar{e}, 0)$, with $\bar{e}$ being its distance from the $x$-axis (see Figure 1).

By denoting by $w=w(s, t)$ the deflection in the weak plane, and by $\theta=\theta(s, t)$ the twist angle, $t$ being the time, the linearized equations of motion of the foil beam, in nondimensional form, read

$$
\begin{array}{r}
-\frac{1}{\Lambda^{2}} \ddot{\theta}-c_{r} \dot{\theta}+\Gamma \theta^{\prime \prime}+\zeta \dot{\theta}^{\prime \prime}=0, \\
\ddot{w}+c_{t} \dot{w}+w^{\prime \prime \prime \prime}+\eta \dot{w}^{\prime \prime \prime \prime}+2 \mu w^{\prime \prime}=0,
\end{array}
$$

in which the dot and the prime symbolize derivatives with respect to the nondimensional time $t$ and abscissa $s$, respectively.

Similarly, the relevant boundary conditions are

$$
\begin{gathered}
\theta_{A}=0, \\
w_{A}=0,
\end{gathered}
$$




$$
\begin{aligned}
w_{A}^{\prime} & =0, \\
\Gamma \theta_{B}^{\prime}+\zeta \dot{\theta}_{B}+k e\left(w_{B}+e \theta_{B}\right)+c_{b} e\left(\dot{w}_{B}+e \dot{\theta}_{B}\right) & =0, \\
-w_{B}^{\prime \prime \prime}-\eta \dot{w}_{B}^{\prime \prime \prime}+k\left(w_{B}+e \theta_{B}\right)+c_{b}\left(\dot{w}_{B}+e \dot{\theta}_{B}\right) & =0, \\
w_{B}^{\prime \prime}+\eta \dot{w}_{B}^{\prime \prime} & =0 .
\end{aligned}
$$

Equations (1) and (2) are obtained by introducing the following positions and by removing the tilde:

$$
\begin{aligned}
& \tilde{t}=\omega t, \\
& \widetilde{s}=\frac{s}{\ell}, \\
& \widetilde{\theta}=\theta, \\
& \widetilde{w}=\frac{w}{\ell}, \\
& \omega=\sqrt{\frac{E J_{y}}{m \ell^{4}}}, \\
& \mu=\frac{F \ell^{2}}{2 E J_{y}}, \\
& e=\frac{\bar{e}}{\ell}, \\
& k=\frac{\bar{k} \ell^{3}}{E J_{y}}, \\
& \Lambda=\sqrt{\frac{m \ell^{2}}{I_{x}}}, \\
& \Gamma=\frac{G J_{x}}{E J_{y}}, \\
& \widetilde{c}_{t}=\frac{c_{t} \omega \ell^{4}}{E J_{y}}, \\
& \tilde{\eta}=\frac{\eta \omega}{E}, \\
& \widetilde{c}_{r}=\frac{c_{r} \omega \ell^{2}}{E J_{y}}, \\
& \tilde{\zeta}=\frac{\zeta \omega J_{x}}{E J_{y}}, \\
& \widetilde{c}_{b}=\frac{c_{b} \omega \ell^{3}}{E J_{y}} \text {. }
\end{aligned}
$$

Finally, it is important to remark that when $e=0$, the flexural motion (in linear regime) is uncoupled from the torsional one.

\section{Linear Stability Analysis}

Stability of the trivial equilibrium position of the beam, namely, $w=\theta=0$, is addressed, with the aim of evaluating the critical value of the follower force at which flutter or divergence bifurcations occur. To this end, an exact analysis of the eigenvalues of the boundary value problems, for both the undamped and damped cases, is developed.

3.1. The Flutter Load of the Undamped Foil Beam. The undamped case $\left(c_{r}=c_{t}=c_{b}=\eta=\xi=0\right)$ is considered first. The relevant problem reads

$$
\begin{aligned}
-\frac{1}{\Lambda^{2}} \ddot{\theta}+\Gamma \theta^{\prime \prime} & =0, \\
\ddot{w}+w^{\prime \prime \prime \prime}+2 \mu w^{\prime \prime} & =0,
\end{aligned}
$$

together with

$$
\begin{aligned}
\theta_{A} & =0, \\
w_{A} & =0, \\
w_{A}^{\prime} & =0, \\
\Gamma \theta_{B}^{\prime}+k e\left(w_{B}+e \theta_{B}\right) & =0, \\
-w_{B}^{\prime \prime \prime}+k\left(w_{B}+e \theta_{B}\right) & =0, \\
w_{B}^{\prime \prime} & =0 .
\end{aligned}
$$

By letting a solution be in the form

$$
\left(\begin{array}{l}
\theta(s, t) \\
w(s, t)
\end{array}\right)=\left(\begin{array}{l}
\widehat{\theta}(s) \\
\widehat{w}(s)
\end{array}\right) \exp (\lambda t),
$$

the following boundary value problem is obtained:

$$
\begin{aligned}
-\frac{\lambda^{2}}{\Lambda^{2}} \widehat{\theta}+\Gamma \widehat{\theta}^{\prime \prime} & =0 \\
\lambda^{2} \widehat{w}+\widehat{w}^{\prime \prime \prime \prime}+2 \mu \widehat{w}^{\prime \prime} & =0 \\
\widehat{\theta}_{A} & =0, \\
\widehat{w}_{A} & =0, \\
\widehat{w}_{A}^{\prime} & =0, \\
\Gamma \widehat{\theta}_{B}^{\prime}+k e\left(\widehat{w}_{B}+e \widehat{\theta}_{B}\right) & =0, \\
-\widehat{w}_{B}^{\prime \prime \prime}+k\left(\widehat{w}_{B}+e \widehat{\theta}_{B}\right) & =0, \\
\widehat{w}_{B}^{\prime \prime} & =0 .
\end{aligned}
$$

The field equations (7a) and (7b) are uncoupled, which makes the problem easy to be solved. In particular, the deflection $\widehat{w}(s)$ coincides with that of the planar Beck's beam (see, e.g., [33]). By taking into account the geometrical boundary 
conditions, (7c), (7d), and (7e), the solution of the field equations can be written as

$$
\begin{aligned}
\widehat{\theta}(s)= & \frac{C_{1}}{\gamma} \sin (\gamma s), \\
\widehat{w}(s)= & C_{2}(\cos (\alpha s)-\cosh (\beta s)) \\
& +C_{3}\left(\frac{1}{\alpha} \sin (\alpha s)-\frac{1}{\beta} \sinh (\beta s)\right),
\end{aligned}
$$

where $C_{j}(j=1, \ldots, 3)$ are arbitrary constants and $\alpha, \beta$, and $\gamma$ are wave-numbers, defined by

$$
\alpha^{2}=\mu+\sqrt{\mu^{2}-\lambda^{2}}
$$

$$
\begin{aligned}
& \beta^{2}=-\mu+\sqrt{\mu^{2}-\lambda^{2}}, \\
& \gamma^{2}=-\frac{\lambda^{2}}{\Lambda^{2} \Gamma} .
\end{aligned}
$$

By replacing (8) in the mechanical boundary conditions of problem with $(7 \mathrm{f}),(7 \mathrm{~g})$, and $(7 \mathrm{~h})$, and rearranging, the following algebraic problem is obtained:

$$
\left[\begin{array}{ll}
\mathbf{B}_{\theta \theta} & \mathbf{B}_{\theta w} \\
\mathbf{B}_{w \theta} & \mathbf{B}_{w w}
\end{array}\right] \mathbf{c}=\mathbf{0},
$$

where $\mathbf{c}:=\left\{C_{j}\right\}^{T}$ is the column vector of the unknown constants, and

$$
\begin{aligned}
\mathbf{B}_{\theta \theta} & :=\Gamma \cos (\gamma)+\frac{e^{2} k}{\gamma} \sin (\gamma) \\
\mathbf{B}_{\theta w}: & {\left[e k(\cos (\alpha)-\cosh (\beta)) e k\left(\frac{\sin (\alpha)}{\alpha}-\frac{\sinh (\beta)}{\beta}\right)\right] } \\
\mathbf{B}_{w \theta}:= & {\left[\begin{array}{ll}
\frac{e k}{\gamma} \sin (\gamma) & 0
\end{array}\right]^{T}, } \\
\mathbf{B}_{w w}:= & {\left[\begin{array}{ccc}
-\alpha^{3} \sin (\alpha)+\beta^{3} \sinh (\beta)+k(\cos (\alpha)-\cosh (\beta)) & \left.\alpha^{2} \cos (\alpha)+\beta^{2} \cosh (\beta)+k\left(\frac{\sin (\alpha)}{\alpha}-\frac{\sinh (\beta)}{\beta}\right)\right] \\
-\alpha^{2} \cos (\alpha)-\beta^{2} \cosh (\beta) & -\alpha \sin (\alpha)-\beta \sinh (\beta)
\end{array}\right] }
\end{aligned}
$$

are matrices depending on the eigenvalue $\lambda$, on the follower force $\mu$, and on the stiffness and eccentricity of the spring, $k$ and $e$, respectively, by the way of wave-numbers $\alpha, \beta$, and $\gamma$. By zeroing the determinant of the $(3 \times 3)$ matrix of coefficients in (10), a transcendental characteristic equation $f_{u}(\lambda ; \mu, k, e)=0$ is obtained. Since the system is undamped, all the eigenvalues lie on the imaginary axis in the precritical phase, that is, when the value of the force is less than the critical one; namely, $\lambda=i \omega$.

For a given set $(k, e)$, the undamped critical flutter load $\mu_{u}$ is found as the lowest value of the force at which a pair of purely imaginary eigenvalues coalesce. By taking $(\mu, k)$ as control parameters and $e$ as an auxiliary parameter (kept fixed), this coalescence mechanism takes place on the manifold $\mathscr{H}^{u}$ (symbol $\mathscr{H}$ denoting Hopf bifurcation):

$$
\mathscr{H}^{u}:=\left\{\begin{array}{l}
f_{u}(\omega ; \mu, k ; e)=0 \\
\frac{\partial f_{u}(\omega ; \mu, k ; e)}{\partial \omega}=0 .
\end{array}\right.
$$

System (12) implicitly defines a multibranch curve in the $(\mu, k)$-parameter plane, parametrized by $\omega$. Only numerical solution can be pursued for this system.

3.2. The Flutter Load of the Damped Foil Beam. When internal and external (distributed and lumped) damping act on the foil beam, the linear problem is governed by the field equations (1), and by the boundary conditions (2). By using the separation of variables (6), the spatial boundary value problem follows:

$$
\begin{aligned}
-\left(\frac{\lambda^{2}}{\Lambda^{2}}+c_{r} \lambda\right) \hat{\theta}+(\Gamma+\zeta \lambda) \widehat{\theta}^{\prime \prime} & =0, \\
\left(\lambda^{2}+c_{t} \lambda\right) \widehat{w}+(1+\eta \lambda) \widehat{w}^{\prime \prime \prime \prime}+2 \mu \widehat{w}^{\prime \prime} & =0, \\
\widehat{\theta}_{A} & =0, \\
\widehat{w}_{A} & =0, \\
\widehat{w}_{A}^{\prime} & =0, \\
(\Gamma+\zeta \lambda) \widehat{\theta}_{B}^{\prime}+e\left(k+c_{b} \lambda\right)\left(\widehat{w}_{B}+e \widehat{\theta}_{B}\right) & =0, \\
-(1+\eta \lambda) \widehat{w}_{B}^{\prime \prime \prime}+\left(k+c_{b} \lambda\right)\left(\widehat{w}_{B}+e \widehat{\theta}_{B}\right) & =0, \\
(1+\eta \lambda) \widehat{w}_{B}^{\prime \prime} & =0,
\end{aligned}
$$

whose solution is still given by (8), but with the wavenumbers and matrices redefined as follows: 


$$
\begin{aligned}
& \alpha^{2}=\frac{\mu+\sqrt{\mu^{2}-\lambda\left(c_{t}+\lambda\right)(1+\eta \lambda)}}{1+\eta \lambda}, \\
& \beta^{2}=\frac{-\mu+\sqrt{\mu^{2}-\lambda\left(c_{t}+\lambda\right)(1+\eta \lambda)}}{1+\eta \lambda}, \\
& \gamma^{2}=-\frac{\lambda^{2} / \Lambda^{2}+c_{r} \lambda}{\Gamma+\zeta \lambda} \\
& \mathbf{B}_{\theta \theta}:=(\Gamma+\zeta \lambda) \cos (\gamma)+\frac{e^{2}\left(k+c_{b} \lambda\right)}{\gamma} \sin (\gamma), \\
& \mathbf{B}_{\theta w}:=\left[e\left(k+c_{b} \lambda\right)(\cos (\alpha)-\cosh (\beta)) e\left(k+c_{b} \lambda\right)\left(\frac{\sin (\alpha)}{\alpha}-\frac{\sinh (\beta)}{\beta}\right)\right] \\
& \mathbf{B}_{w \theta}:=\left[\frac{e\left(k+c_{b} \lambda\right)}{\gamma} \sin (\gamma) 0\right]^{T}, \\
& \mathbf{B}_{w w} \quad-(1+\eta \lambda)\left(\alpha^{2} \cos (\alpha)+\beta^{2} \cosh (\beta)\right) \\
& :=\left[\begin{array}{r}
\left.-(1+\eta \lambda)\left(\alpha^{3} \sin (\alpha)-\beta^{3} \sinh (\beta)\right)+\left(k+c_{b} \lambda\right)(\cos (\alpha)-\cosh (\beta))(1+\eta \lambda)\left(\alpha^{2} \cos (\alpha)+\beta^{2} \cosh (\beta)\right)+\left(k+c_{b} \lambda\right)\left(\frac{\sin (\alpha)}{\alpha}-\frac{\sinh (\beta)}{\beta}\right)\right]
\end{array}\right.
\end{aligned}
$$

To compute the (damped) flutter load $\mu_{d}$, the following algorithm is applied: (i) the characteristic equation $f_{d}\left(\lambda, \mu, k, e ; c_{r}, c_{t}, c_{b}, \zeta, \eta\right)=0$, now depending on three external and two internal damping coefficients, is obtained; (ii) $\lambda=\xi+i \omega$ is put in this equation and its real and imaginary parts are separated, thus obtaining

$$
\begin{aligned}
& f_{d}\left(\lambda, \mu, k, e ; c_{r}, c_{t}, c_{b}, \zeta, \eta\right) \\
&:= f\left(\xi, \omega ; \mu, k, e ; c_{r}, c_{t}, c_{b}, \zeta, \eta\right) \\
&+i g\left(\xi, \omega ; \mu, k, e ; c_{r}, c_{t}, c_{b}, \zeta, \eta\right)=0,
\end{aligned}
$$

with $f, g \in \mathbb{R}$; (iii) $\xi=0$ is taken, since at the critical flutter load a single pair of eigenvalues crosses (from the left) the imaginary axis (simple Hopf bifurcation); (iv) for a given set of parameters $\left(k, e, c_{r}, c_{t}, c_{b}, \zeta, \eta\right)$, the system $f=0, g=0$ is solved for the two unknowns $\mu$ and $\omega$, by looking for the lowest $\operatorname{root} \mu=\mu_{d}$.

Finally, by still taking $(\mu, k)$ as control parameters and considering all the remaining ones as (fixed) auxiliary parameters, the (damped) flutter mechanism takes place on the manifold $\mathscr{H}^{d}$ :

$$
\mathscr{H}^{d}:=\left\{\begin{array}{l}
f\left(0, \omega ; \mu, k ; e, c_{r}, c_{t}, c_{b}, \zeta, \eta\right)=0 \\
g\left(0, \omega ; \mu, k ; e, c_{r}, c_{t}, c_{b}, \zeta, \eta\right)=0 .
\end{array}\right.
$$

This equation implicitly defines a multibranch curve in the $(\mu, k)$-parameter plane, parametrized by $\omega$. No closed-form solutions, but only numerical, can be pursued for system (16).

3.3. The Divergence Load of the Foil Beam. In order to find the divergence boundary, the locus $\mathscr{D}$ of the roots $\xi=0, \omega=0$ of the characteristic equation must be found. As done above, the locus can be determined by solving the system:

$$
\mathscr{D}:=\left\{\begin{array}{l}
f\left(0,0 ; \mu, k ; e, c_{r}, c_{t}, c_{b}, \zeta, \eta\right)=0 \\
g\left(0,0 ; \mu, k ; e, c_{r}, c_{t}, c_{b}, \zeta, \eta\right)=0 .
\end{array}\right.
$$

It is found that the second equation of system (17) identically vanishes for any set of parameters, while the first equation of system (17) is independent of the damping coefficients. A closed-form solution can be found for system (17); namely,

$$
\begin{aligned}
\mathscr{D}:= & 4 \mu^{2}\left(\Gamma+e^{2} k\right)-2 \Gamma k \mu \cos (\sqrt{2 \mu}) \\
& +\Gamma k \sqrt{2 \mu} \sin (\sqrt{2 \mu})=0
\end{aligned}
$$

This equation implicitly defines a multibranch curve in the $(\mu, k)$-parameter plane.

\section{Numerical Results}

Numerical analyses are here referred to as a foil beam, having a rectangular cross-section, of width $b$ and thickness $h$ (see Figure 1). Accordingly, the following (dimensional) relations hold: $J_{y}=(1 / 12) b h^{3}, J_{x}=(1 / 3) b h^{3}, m:=b h \rho$, and $I_{x}=$ $(1 / 12) b^{3} h \rho$, with $\rho$ being the mass density.

An exhaustive analysis of all parameters would be cumbersome. Therefore, the following assumptions, linking external and internal damping coefficients, are made: (i) linear distribution of local damping forces, due to a uniform disposition of external dashpots on the cross-section; (ii) negligible material bulk viscous deformation. Accordingly, 
the relevant dimensional damping coefficients satisfy the following relations:

$$
\begin{aligned}
c_{r} & =\frac{1}{12} b^{2} c_{t}, \\
\zeta & =\frac{1}{3} \eta .
\end{aligned}
$$

Moreover, a length-to-width ratio equal to 5 is considered, for which the following nondimensional quantities read

$$
\begin{aligned}
& \Lambda=10 \sqrt{3}, \\
& \Gamma=\frac{2}{1+\nu}, \\
& c_{r}=\frac{c_{t}}{300}, \\
& \zeta=\frac{4}{3} \eta,
\end{aligned}
$$

with $v$ being the Poisson coefficient, which has been taken equal to 0.3 . In addition, the chosen numerical values for the damping coefficients are $c_{t}=1 / 10, c_{b}=1 / 10$, and $\eta=1 / 100$.

The following case studies, relevant to two different positions of the spring-damper device, are examined:

(i) case study I: the foil cantilever beam is braced at the tip, without eccentricity; namely, $\bar{e}=0$.

(ii) case study II: the foil cantilever beam is braced at the tip, with the spring-damper device located the maximum distance from the $z$-axis; namely, $\bar{e}=b / 2$.

Undamped Foil Beam. The linear stability diagram of the undamped foil beam is displayed in Figure 2. Here, the critical load $\mu$ is plotted versus the stiffness of the spring $k$, for the case studies I (black curves) and II (gray curves), respectively. For each of the case studies, two curves are shown in the figure: (i) $\mathscr{D}$ is the divergence locus, at which the straight configuration loses stability via a static bifurcation, which, as discussed above, is independent of damping; (ii) $\mathscr{H}^{u}$ is the undamped Hopf locus, at which the foil beam loses stability via a dynamic bifurcation (collision of two pairs of eigenvalues).

When $k=0$, the critical flutter load of the undamped Beck's beam is recovered; namely, $\mu_{u}=10.02$ (see, e.g., $[24,28])$. When $\mu$ is increased from 0 , it is apparent that the braced foil beam can exploit two different mechanisms of bifurcations, depending on the magnitude of $k$, namely, a dynamic one (curve $\mathscr{H}^{u}$ ) for small values of $k$, and a static one (curve $\mathscr{D}$ ) for large values of $k$. The phenomenon can be explained by the fact that when $k \rightarrow \infty$, the spring becomes a fix support, rendering the system conservative, since the transverse component of the follower forces cannot expend any work on the transverse displacement $w_{B}=0$ (see also [22] for additional references). The two curves meet tangentially at a (degenerate) double-zero point, at which the two imaginary eigenvalues of the Hopf bifurcation collide at the zero frequency.

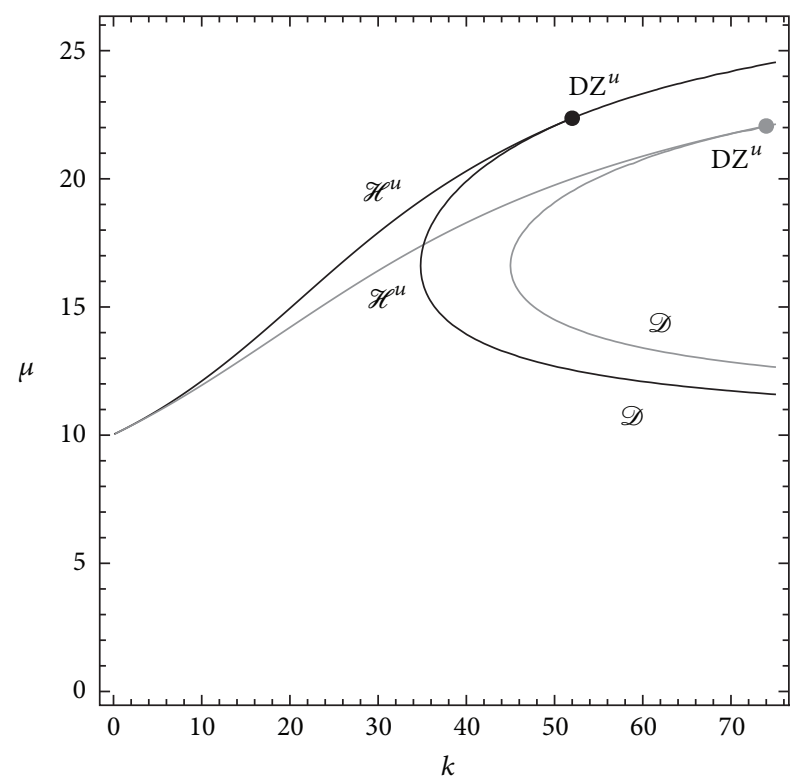

FIGURE 2: Linear stability diagram of the undamped foil beam in the $(\mu, k)$-plane, when the length-to-width-ratio is equal to 5 . Black curves: case study I $(e=0)$. Gray curves: case study II $(e=1 / 10)$. $\mathscr{H}^{u}$ : undamped Hopf locus, $\mathscr{D}$ : divergence locus, and DZ: doublezero bifurcation point.

In conclusion, it is proved that the spring has a beneficial effect on the flutter behavior of the undamped Beck's beam, by increasing $\mu_{u}$ up to about two times in the dynamic bifurcation range (case I). This is due to the fact that the spring increases the distance among the natural frequencies of the unloaded beam, and, therefore, it delays their collision, caused by an increasing of the follower force. On the contrary, when case study II is addressed, it is found that the flexuraltorsional coupling, due to the eccentricity of the spring, is detrimental on the dynamic bifurcation, since it lowers the Hopf curve; as a consequence, the best location for the spring is $e=0$. However, the coupling is beneficial on the static bifurcation, since now this bifurcation occurs at larger $k$ values, and the (significant) lower branch of the $\mathscr{D}$-locus is above that of case study I; consequently, the maximum eccentricity is the optimum for increasing the bifurcation static load.

It is worth noticing that the effect of the flexural-torsional coupling strongly depends on the length-to-width ratio; indeed, the larger this ratio is, the smaller the difference between the two case studies is. In order to show this outcome, the linear stability diagram, which corresponds to a length-to-width ratio equal to 10 , namely, $\Lambda=20 \sqrt{3}$, is shown in Figure 3.

Damped Foil Beam. The linear stability diagram relevant to the damped foil beam is displayed in Figure 4, for case studies I (Figure 4(a)) and II (Figure 4(b)), respectively. It is seen that, while the divergence locus is not changed by damping, the Hopf curves, at which a generic dynamic bifurcation occurs, determined by the crossing of the imaginary axis of one pair of eigenvalues, are affected by the damping parameters. The 


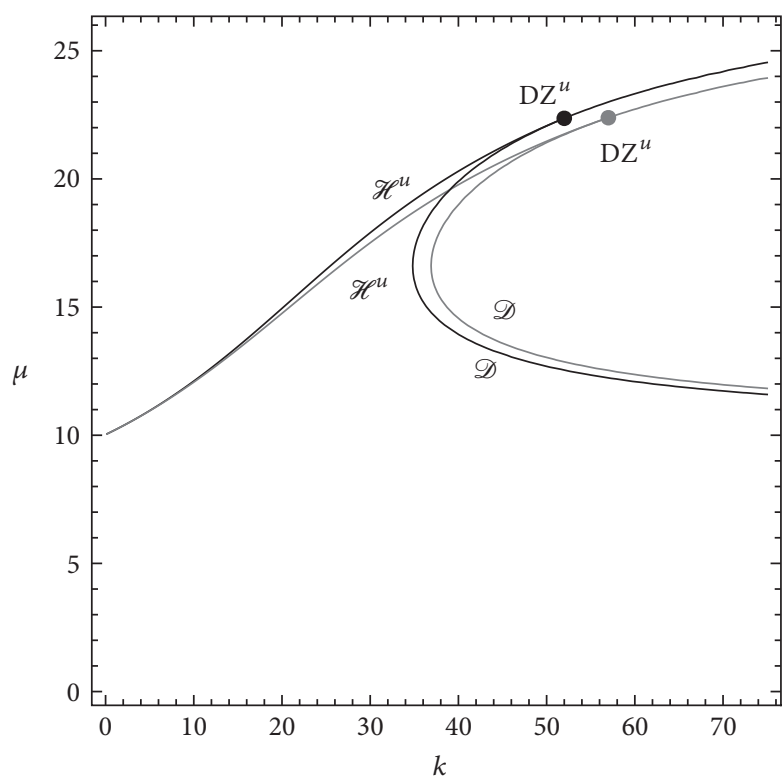

FIGURE 3: Linear stability diagram of the undamped foil beam in the $(\mu, k)$-plane, when the length-to-width-ratio is equal to 10 . Black curves: case study I $(e=0)$. Gray curves: case study II $(e=1 / 20)$. $\mathscr{H}^{u}$ : undamped Hopf locus, $\mathscr{D}$ : divergence locus, and DZ: doublezero bifurcation point.

plots are obtained by considering different damping forms, in the numerical simulations, namely: (i) lumped dashpot at the tip (labels of curves and points marked with the apex d1); (ii) distributed internal and external damping (labels of curves and points marked with the apex $d 2$ ); (iii) all the damping forms acting simultaneously (labels of curves and points marked with the apex $d 3$ ). Moreover, the curves referred to as damped beams are denoted with black lines in Figure 4, while the gray ones are relevant to the undamped foil beam.

The scenario discussed in Figure 2 abruptly changes, when a dashpot is added at the tip (changing the Hopf curve $\mathscr{H}^{u}$ into $\mathscr{H}^{d 1}$ ). Irrespectively of the magnitude of the damping parameter $c_{b}$, the Hopf curve is below the divergence curve for all values of $k$, and for both the case studies. Therefore, due to presence of the dashpot, dynamic instability occurs also for large values of $k$. The destabilizing effect of damping is apparent (even if just lumped at the tip): it entails $\mu_{d}<\mu_{u}$ not only at $k=0$, but at any $k$. Remarkably, the flexural-torsional coupling has not effects on this behavior.

When distributed internal and external damping are considered (changing the Hopf curve $\mathscr{H}^{u}$ into $\mathscr{H}^{d 2}$ ), the well known destabilization paradox is encountered $[8,9,24]$. The detrimental effect of damping on the undamped beam is confirmed at small values of $k$, although it depends on the ratio between the damping parameters $c_{t}$ and $\eta$; it is worth noticing that an interval of values of $k$ can, in principle, exist, for which distributed damping produces, instead, a beneficial effect. Again, the flexural-torsional coupling does not change this behavior.

It is important to remark that the destabilization phenomenon is triggered in Beck's column by internal damping
[5], that is, by a form of dissipation depending on the viscoelastic (Kelvin-Voigt) properties of the column, which is, indeed, proportional to the stiffness distribution; external damping, that is, dissipation proportional to the mass distribution, is, instead, stabilizing. It is worth noticing that Beck's column is to be considered a particular case in this context, since, in general elastic systems, loaded by nonconservative and configuration-dependent loads, stabilizing damping is sophisticatedly related to both the mass and stiffness distribution [42]; as a matter of fact, while the air drag is stabilizing for Beck's column, it is, instead, destabilizing for Pflüger's column [43], namely, a Beck's beam with a different mass distribution, that is, with an added point mass at the loaded end.

When, however, a dashpot is added at the tip of an internally and externally damped beam (changing the Hopf curve $\mathscr{H}^{d 2}$ into $\mathscr{H}^{d 3}$ ), it has a beneficial effect when $k$ is small, by partially counteracting the destabilization paradox, notwithstanding the fact that it is not sufficient to bring the load at $\mu_{u}$ when $k=0$. When $k$ is sufficiently large, this beneficial effect ends, and the external dashpot becomes detrimental, since it renders $\mathscr{H}^{d 3}$ lower than $\mathscr{H}^{d 2}$. As before, the flexural-torsional coupling is not able to qualitatively change the behavior of the damped planar beam.

Finally, the critical manifold defined by (16), which is a hypersurface in the $\left(\mu, k, e, c_{r}, c_{t}, c_{b}, \zeta, \eta\right)$-parameter space, can be conveniently represented by taking $\left(\eta, c_{t}\right)$ as control parameters and the remaining ones as (fixed) auxiliary parameters and by performing sections at $\mu=$ const. The correspondent $\mu$-isolines of the foil beam's critical manifold, when $c_{b}=0$, are displayed in Figure 5, for the unbraced beam (continuous black curves), for case study I (dashed black curves) and for case study II (continuous gray curves), respectively. It is found that the presence of the spring enlarges the stable region, since it moves to the left of the $\mu$-isolines of the unbraced beam. However, for the selected value of $k$, namely, $k=1$, there is no significant difference between the two case studies, as it is also confirmed by the damped Hopf curves in Figures 4(a) and 4(b), which, indeed, are nearly coincident when $k$ is small.

\section{Conclusions}

The flutter and buckling behavior of a 3D cantilever foil beam, loaded at the tip by a follower force, internally and externally damped, have been investigated in this paper. The role of different forms of damping, distributed and lumped, as well as of the flexural-torsional coupling, has been explored. The latter has been triggered by a linear spring-damper device, located in an eccentric position with respect to beam axis.

The linearized equations of motion of the system have been recalled. Then, the relevant linear stability diagrams have been built up via an exact analysis of the eigenvalues of the associated boundary value problem.

In particular, the following conclusions can be drawn.

(1) The flexural-torsional coupling has a destabilizing effect on the dynamic stability of both damped and undamped beams. 


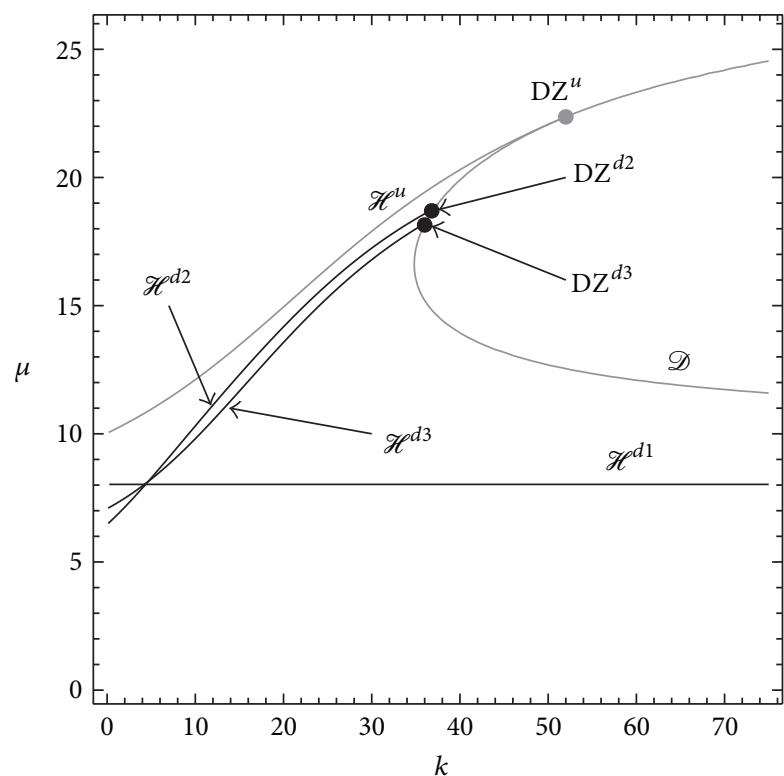

(a)

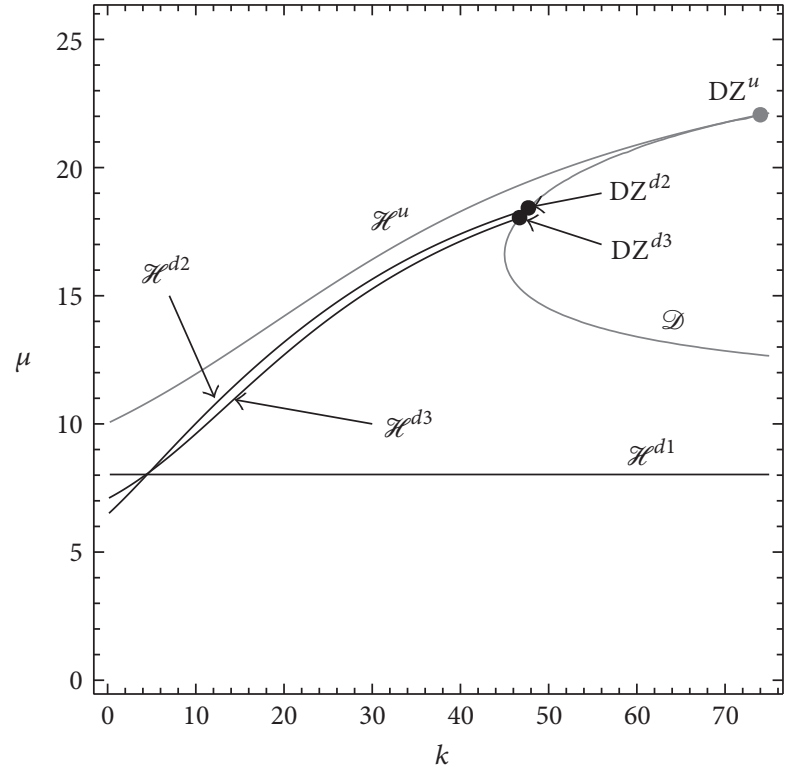

(b)

FIGURE 4: Linear stability diagrams of the damped foil beam in the $(\mu, k)$-plane: (a) case study I; (b) case study II. Black curves: damped beam. Gray curves: undamped beam. $\mathscr{H}^{u}$ : undamped Hopf locus, $\mathscr{H}^{d 1}$ : Hopf locus for lumped dashpot at the tip, $\mathscr{H}^{d 2}$ : Hopf locus for distributed internal and external damping, $\mathscr{H}^{d 3}$ : Hopf locus for all the damping forms acting simultaneously, $\mathscr{D}$ : divergence locus, and DZ: double-zero bifurcation point.

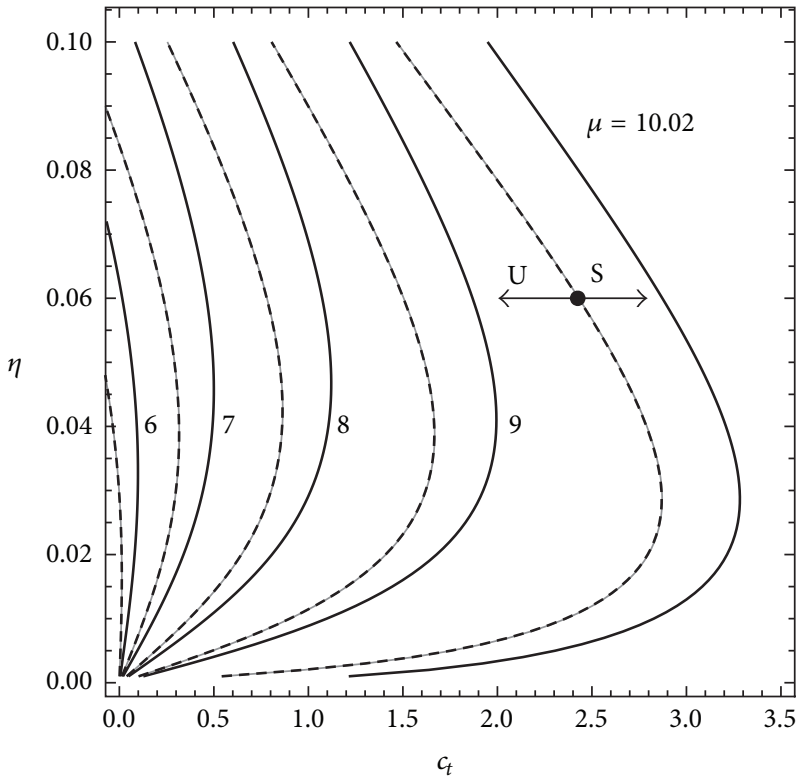

Figure 5: $\mu$-isolines in the $\left(\eta, c_{t}\right)$-plane, when $k=1$. Continuous black curves, unbraced beam. Dashed black curves, case study I. Continuous gray curves, case study II. U: unstable region, S: stable region.

(2) The flexural-torsional coupling is stabilizing for the static bifurcation.

(3) The stabilizing and destabilizing effects of the flexural-torsional coupling are reduced when the lengthto-width-ratio is increased.
(4) The lumped dashpot at the tip has a detrimental effect on the dynamic stability; this effect does not depend on the flexural-torsional coupling; that is, it holds for any stiffness and eccentricity of the lumped springdamper device.

(5) The effect of coupling does not qualitatively change the dynamic stability of damped beams.

\section{Conflicts of Interest}

The authors declare that there are no conflicts of interest regarding the publication of this paper.

\section{References}

[1] W. Koiter, "Unrealistic follower forces," Journal of Sound and Vibration, vol. 194, no. 4, p. 636, 1996.

[2] Y. Sugiyama, M. A. Langthjem, and B.-J. Ryu, "Realistic follower forces," Journal of Sound and Vibration, vol. 225, no. 4, pp. 779782, 1999.

[3] H. Ziegler, "Die Stabilitätskriterien der Elastomechanik," Ingenieur-Archiv, vol. 20, no. 1, pp. 49-56, 1952.

[4] M. Beck, "Die Knicklast des einseitig eingespannten, tangential gedrückten Stabes," ZAMP Zeitschrift für angewandte Mathematik und Physik, vol. 3, no. 3, pp. 225-228, 1952.

[5] V. V. Bolotin, Nonconservative Problems of the Theory of Elastic Stability, Corrected and authorized edition. Translated from the Russian by T. K. Lusher. English translation edited by G. Herrmann, A Pergamon Press Book. The Macmillan Co., New York, 1963. 
[6] G. Herrmann, "Stability of equilibrium of elastic systems subjected to non-conservative forces," Applied Mechanics Reviews, vol. 20, pp. 103-108, 1967.

[7] I. P. Andreichikov and V. I. Yudovich, "The stability of viscoelastic rods," Izvestiya Akad Nauk SSSR Mekhanika Tverdogo Tela, vol. 9, no. 2, pp. 78-87, 1974.

[8] A. P. Seyranian and A. A. Mailybaev, Multiparameter Stability Theory with Mechanical Applications, vol. 13 of Series on Stability, Vibration and Control of Systems. Series A: Textbooks, Monographs and Treatises, World Scientific Publishing Co., Inc, Singapore, Singapore, 2003.

[9] O. N. Kirillov, Nonconservative stability problems of modern physics, vol. 14 of De Gruyter Studies in Mathematical Physics, De Gruyter, Berlin, 2013.

[10] M. A. Langthjem and Y. Sugiyama, "Dynamic stability of columns subjected to follower loads: a survey," Journal of Sound and Vibration, vol. 238, no. 5, pp. 809-851, 2000.

[11] K. Ingerle, "Stability of massless non-conservative elastic systems," Journal of Sound and Vibration, vol. 332, no. 19, pp. 45294540, 2013.

[12] D. Bigoni and G. Noselli, "Experimental evidence of flutter and divergence instabilities induced by dry friction," Journal of the Mechanics and Physics of Solids, vol. 59, no. 10, pp. 2208-2226, 2011.

[13] I. Elishakoff, "Controversy associated with the so-called 'follower forces': critical overview," Applied Mechanics Reviews, vol. 58, no. 2, pp. 117-142, 2005.

[14] B. J. Ryu and Y. Sugiyama, "Dynamic stability of cantilevered timoshenko columns subjected to a rocket thrust," Computers and Structures, vol. 51, no. 4, pp. 331-335, 1994.

[15] A. Mazidi, S. A. Fazelzadeh, and P. Marzocca, "Flutter of aircraft wings carrying a powered engine under roll maneuver," Journal of Aircraft, vol. 48, no. 3, pp. 874-883, 2011.

[16] H. Troger and A. Steindl, Nonlinear stability and bifurcation theory, Springer-Verlag, Vienna, 1991.

[17] M. P. Païdoussis and N. T. Issid, "Dynamic stability of pipes conveying fluid," Journal of Sound and Vibration, vol. 33, no. 3, pp. 267-294, 1974.

[18] L. Wang, "Flutter instability of supported pipes conveying fluid subjected to distributed follower forces," Acta Mechanica Solida Sinica, vol. 25, no. 1, pp. 46-52, 2012.

[19] J. E. Mottershead, "Vibration- and friction-induced instability in disks," Shock and Vibration Digest, vol. 30, no. 1, pp. 14-31, 1998.

[20] N. M. Kinkaid, O. M. O’Reilly, and P. Papadopoulos, "Automotive disc brake squeal," Journal of Sound and Vibration, vol. 267, no. 1, pp. 105-166, 2003.

[21] A. Paolone, M. Vasta, and A. Luongo, "Flexural-torsional bifurcations of a cantilever beam under potential and circulatory forces I: Non-linear model and stability analysis," International Journal of Non-Linear Mechanics, vol. 41, no. 4, pp. 586-594, 2006.

[22] A. Di Egidio, A. Luongo, and A. Paolone, "Linear and nonlinear interactions between static and dynamic bifurcations of damped planar beams," International Journal of Non-Linear Mechanics, vol. 42, no. 1, pp. 88-98, 2007.

[23] A. Luongo and F. D'Annibale, "Double zero bifurcation of non-linear viscoelastic beams under conservative and non-conservative loads," International Journal of Non-Linear Mechanics, vol. 55, pp. 128-139, 2013.

[24] A. Luongo and F. D'Annibale, "On the destabilizing effect of damping on discrete and continuous circulatory systems,"
Journal of Sound and Vibration, vol. 333, no. 24, pp. 6723-6741, 2014.

[25] H. Leipholz, "Über den Einfluß der Dämpfung bei nichtkonservativen Stabilitätsproblemen elastischer Stäbe," IngenieurArchiv, vol. 33, no. 5, pp. 308-321, 1964.

[26] R. H. Plaut, "A New Destabilization Phenomenon in Nonconservative Systems," ZAMM - Journal of Applied Mathematics and Mechanics, vol. 51, no. 4, pp. 319-321, 1971.

[27] P. Hagedorn, "On the destabilizing effect of non-linear damping in non-conservative systems with follower forces," International Journal of Non-Linear Mechanics, vol. 5, no. 2, pp. 341-358, 1970.

[28] O. N. Kirillov and A. P. Seyranian, "The effect of small internal and external friction on the stability of distributed nonconservative systems," Journal of Applied Mathematics and Mechanics, vol. 69, no. 4, pp. 584-611, 2005.

[29] O. N. Kirillov and F. Verhulst, "Paradoxes of dissipationinduced destabilization or who opened Whitney's umbrella?" ZAMM Zeitschrift für Angewandte Mathematik und Mechanik, vol. 90, no. 6, pp. 462-488, 2010.

[30] Y. G. Panovko and S. V. Sorokin, "Quasi-stability of viscoelastic systems with tracking forces," Mechanics of solids, vol. 22, no. 5, pp. 128-132, 1987.

[31] N. Zhinzher, "Influence of dissipative forces with incomplete dissipation on the stability of elastic systems," Mechanics of Solids, vol. 29, no. 1, pp. 135-141, 1994.

[32] A. Luongo and F. D’Annibale, "A paradigmatic minimal system to explain the Ziegler paradox," Continuum Mechanics and Thermodynamics, vol. 27, no. 1-2, pp. 211-222, 2015.

[33] F. D'Annibale, M. Ferretti, and A. Luongo, "Improving the linear stability of the Beck's beam by added dashpots," International Journal of Mechanical Sciences, vol. 110, pp. 151-159, 2016.

[34] M. Pignataro, N. Rizzi, and A. Luongo, Stability and Postcritical Behaviour of Elastic Structures, Elsevier Science Publishers, Amsterdam, Netherlands, 1990.

[35] N. S. Trahair, Flexural-Torsional Buckling of Structures, vol. 6, CRC Press, Boca Raton, Fla, USA, 1993.

[36] D. H. Hodges and G. A. Pierce, "Introduction to structural dynamics and aeroelasticity, second edition," Introduction to Structural Dynamics and Aeroelasticity, Second Edition, pp. 1247, 2011.

[37] W. T. Feldt and G. Herrmann, "Bending-torsional flutter of a cantilevered wing containing a tip mass and subjected to a transverse follower force," Journal of the Franklin Institute, vol. 297, no. 6, pp. 467-478, 1974.

[38] D. H. Hodges, "Lateral-torsional flutter of a deep cantilever loaded by a lateral follower force at the tip," Journal of Sound and Vibration, vol. 247, no. 1, pp. 175-183, 2001.

[39] F. M. Detinko, "Some phenomena for lateral flutter of beams under follower load," International Journal of Solids and Structures, vol. 39, no. 2, pp. 341-350, 2001.

[40] S. A. Fazelzadeh, A. Mazidi, and H. Kalantari, "Bendingtorsional flutter of wings with an attached mass subjected to a follower force," Journal of Sound and Vibration, vol. 323, no. 1-2, pp. 148-162, 2009.

[41] A. Luongo and D. Zulli, Mathematical Models of Beams and Cables, John Wiley Sons, Inc., Hoboken, NJ, USA, 2013.

[42] O. N. Kirillov and A. P. Seyranian, "Stabilization and destabilization of a circulatory system by small velocity-dependent forces," 
Journal of Sound and Vibration, vol. 283, no. 3-5, pp. 781-800, 2005.

[43] M. Tommasini, O. N. Kirillov, D. Misseroni, and D. Bigoni, "The destabilizing effect of external damping: Singular flutter boundary for the Pflüger column with vanishing external dissipation," Journal of the Mechanics and Physics of Solids, vol. 91, pp. 204-215, 2016. 


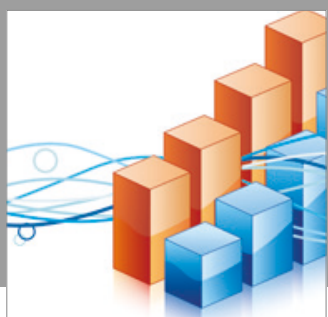

Advances in

Operations Research

vatersals

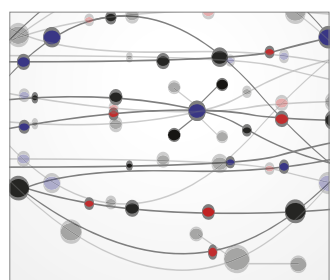

\section{The Scientific} World Journal
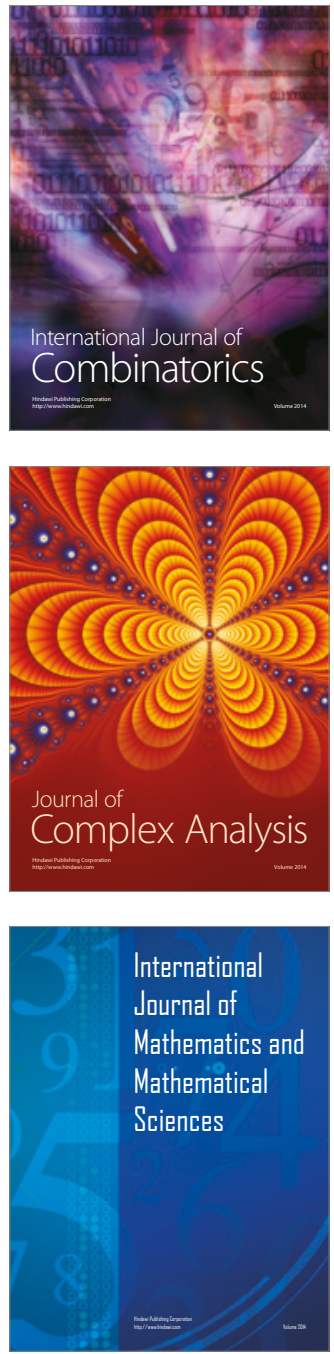
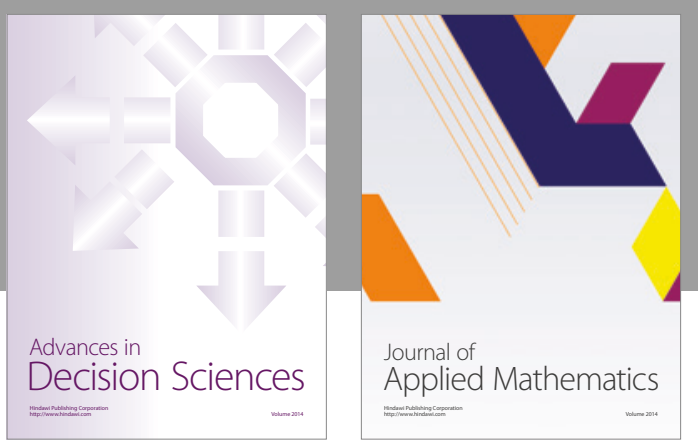

Algebra

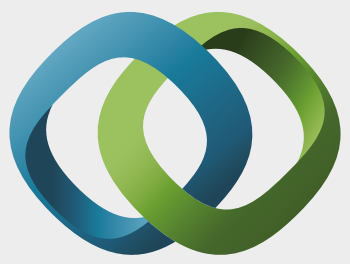

\section{Hindawi}

Submit your manuscripts at

https://www.hindawi.com
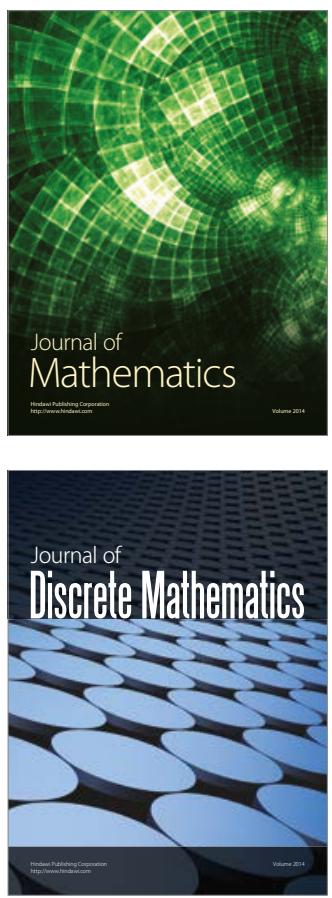

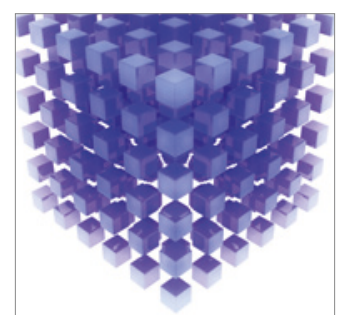

Mathematical Problems in Engineering
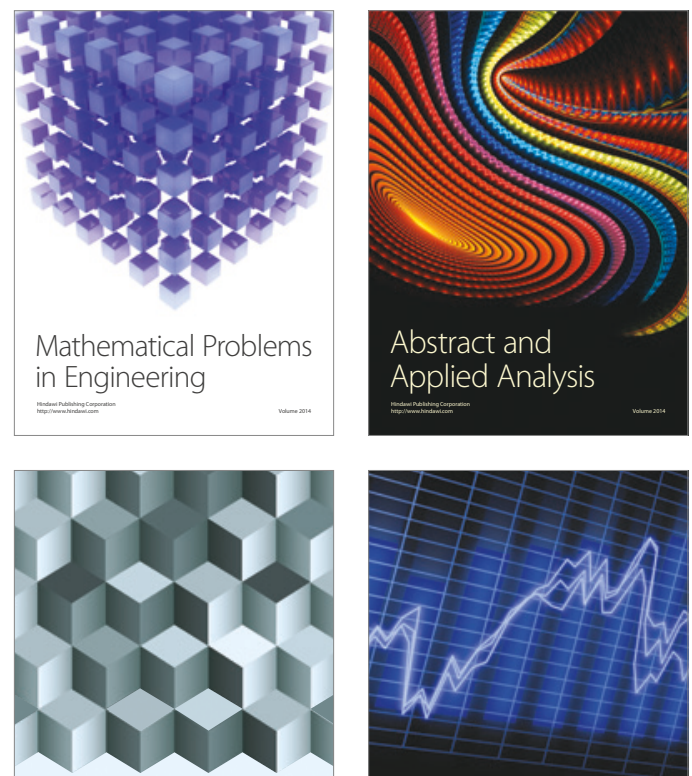

Journal of

Function Spaces

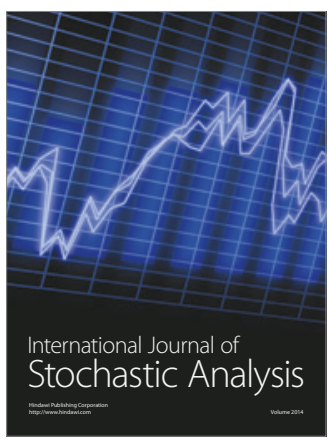

Probability and Statistics
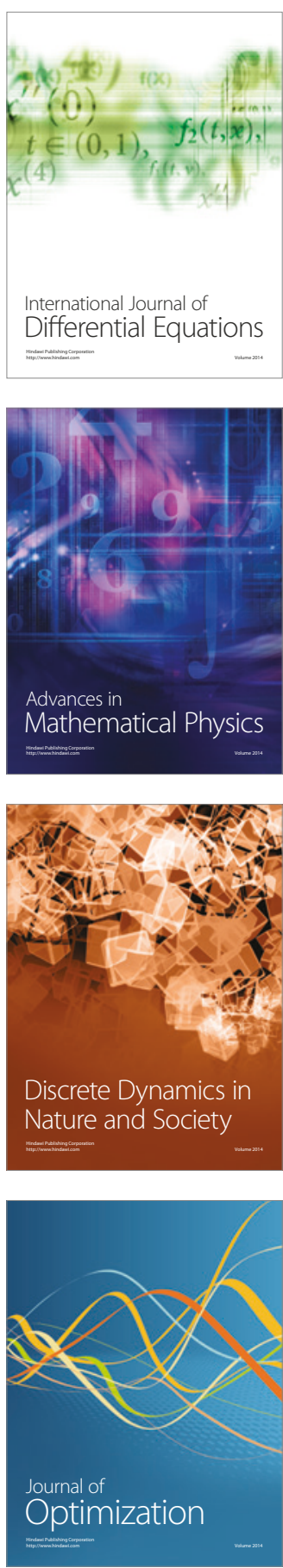